\title{
RFID Array Probe for EM-Field Measurements
}

\author{
S. Capdevila*1, L. Jofre ${ }^{1}$, J.Ch. Bolomey ${ }^{2}$, and J. Romeu ${ }^{1}$ \\ ${ }^{1}$ AntennaLab, Universitat Politècnica de Catalunya. Barcelona, Spain \\ e-mail: scapdevila@tsc.upc.edu \\ ${ }^{2}$ SUPELEC, Electromagnetics Department, Gif-sur-Yvette, France
}

\section{Introduction}

There is a growing need for sensors able to measure or sense the electromagnetic field distribution in a given object, inside or in the surrounding area. Many of the known techniques used in the Industrial or Medical environments use the field scattered by the objects in order to obtain information about the objects that caused them; in most of the cases this characterization is done by measuring in a set of accessible points, where it is possible to use conventional RF probes. With the drawbacks that such probes usually require of bulky connections for the RF feeding, and that such measurements are not always possible if we are interested in measuring the fields inside of a body. An alternative approach to measure the field distribution is by measuring the scattered reflection by well characterized RF probes, what is known as the Modulated Scatterer Technique, [1]. This kind of characterization usually requires the powering cable in order to allow the probes to function, but those are less bulky than RF feeding lines, and can be easily substituted by an external battery.

In this paper we propose a new a solution consisting on the use of an array of RFID-based probes in order to measure the field distribution. The main goal is to demonstrate the capability for the different tags to retrieve the field distribution of a horn antenna in presence of other tags, as a first step towards a complete field measurement where each tag will give the field relative to the rest of the tags in the array.

\section{Near-Field probing formulation}

Figure 1 presents the scenario of operation of a common EM-field probing system. We have an antenna illuminating a certain area, and we are interested in obtaining the field at some specific point. The idea consists on placing an RFID probe at each position we are interested in characterizing and then extract information about the field, in both magnitude and phase, from the signal backscattered by the RFID probe. The information of the EM-field can be extracted by means of the modulated scattering technique (MST), [1]. The reflected signal that can be measured at the transmitting antenna has several components:

$$
b=a \cdot S_{11}+b_{b g n d}+b_{j}
$$

being $S_{11}$ the self-reflection of the antenna, $b_{b g n d}$ unwanted reflections coming from the background and $b_{j}$ the desired information, which depends on the loading of the probe antenna. It can be proved, [2], that the differential reflection coefficient at 


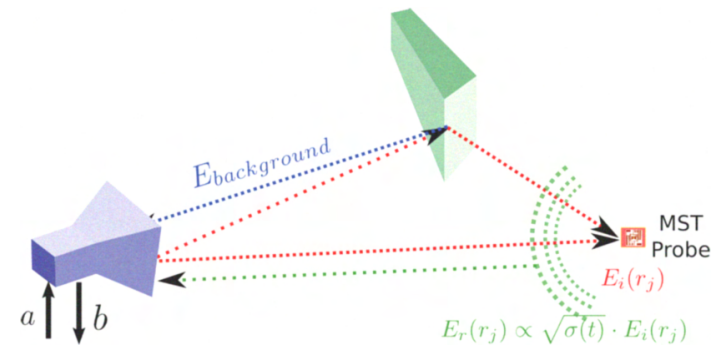

Figure 1: Common scenario of operation of MST-like near-field measurement

the input will be proportional to the differential complex reflection coefficient of the two state-loads and the RFID antenna:

$$
\Delta \rho_{t}\left(r_{j}\right)=\frac{Z_{t r}\left(r_{j}\right)}{2 R_{T}} \underbrace{\left(\tilde{\rho}_{L 1}-\tilde{\rho}_{L 2}\right)}_{\Delta \tilde{\rho}} \propto \sqrt{P_{i}} L_{t}^{2} \Delta \sqrt{\sigma_{L}}
$$

where $Z_{t r}$ is the transfer impedance which accounts for any interaction of the field with the media and offers a general expression for both near and far-field formulation; it can be proved that in far-field a similar expression using the radar cross section of the RFID probe $\left(\sigma_{L}\right)$ can be used, as shown in the right hand of $(2)$, where $L_{t}$ accounts for the losses due to propagation between the antenna and the probe. Due to the powerless nature of passive RFID tags, there is a rectifying circuit at the input of the RFID IC and therefore a non-linear dependence of its input impedance with respect to the power. This non-linearity makes the term $Z_{t r}$ (or $\Delta \sqrt{\sigma_{L}}$ in far field formulation) variable and it needs to be corrected as shown in [3] in order to retrieve a valid field distribution:

$$
\frac{\Delta b\left(P_{r e f}, r_{j}\right.}{\Delta b\left(P_{r e f}, r_{r e f}\right)}=\frac{L_{t r}^{2}\left(r_{j}\right)}{L_{t r}^{2}\left(r_{r e f}\right)} \cdot S\left(\frac{L_{t r}^{2}\left(r_{j}\right)}{L_{t r}^{2}\left(r_{r e f}\right)}\right)
$$

where $S$ is the calibration curve that shows the relationship of the reflected signal with respect to the power.

\section{Experimental setup}

The measurement will be done using 4 Alien ALN 9529 RFID tags. They are placed on a foam support over a line with a separation of $4 \mathrm{~cm}$, see figure 2 . The foam is fixed in a motorized rail that will move the array in front of the measured antenna. The field distribution that is measured is at the $19 \mathrm{~cm} \times 27 \mathrm{~cm}$ aperture of a ridged horn antenna that is placed at about $1 \mathrm{~cm}$ distance of the array, in order to obtain best SNR.

The different tags are successively selected by a SELECT signal with each RFID TAG code generated using a Rohde \& Schwarz SMJ100A Vector Signal Generator; the individual response of the tag is captured coherently using a Rohde \& Schwarz FSL6 spectrum analyzer; figure 3 shows a sketch of the measurement setup.

In order to perform the calibration procedure two sets of measurements are done, the first captures the response of the tag for a power sweep, and the second captures 


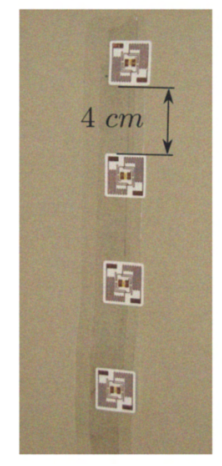

Figure 2: RFID tags array

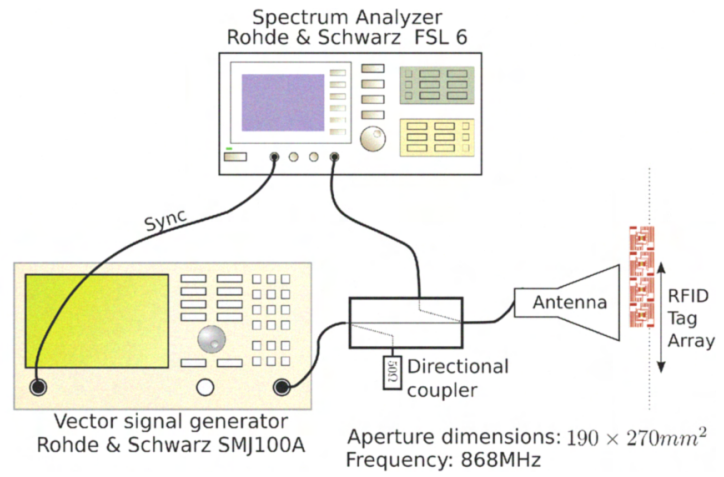

Figure 3: Measurement Setup

the response for a given power and for different positions of the motor. The postprocessing applied to the captured signal consists in identify the RFID tag response, compute the differential signal from both states. By doing so for a set of power levels we obtain the calibration curve that will be used along with the equation (3) to obtain the real field distribution measured by each tag.

\section{Results}

The electric field distribution at the aperture of the horn has been measured along its $\mathrm{H}$-plane at $868 \mathrm{MHz}$. The captured data for each position of the array and for each individual tag has been processed and calibrated as explained before and the relative field distribution for each tag has been plotted; figure 4 shows the obtained field distribution for each of the tags. We can observe that for each of the tags we obtain the same field distribution displaced by the distance between tags; this result shows that the RFID tags in this array configuration do not present high coupling, and therefore each tags is effectively retrieving the field distribution at its position; in order to validate that the shapes for the field distribution are as they should, a simulation of the horn antenna with a Method of Moments solver has been done, and it is shown in figure 5 compared with the distribution of each tag (the individual response for each tag has been centered to allow an easier viewing)

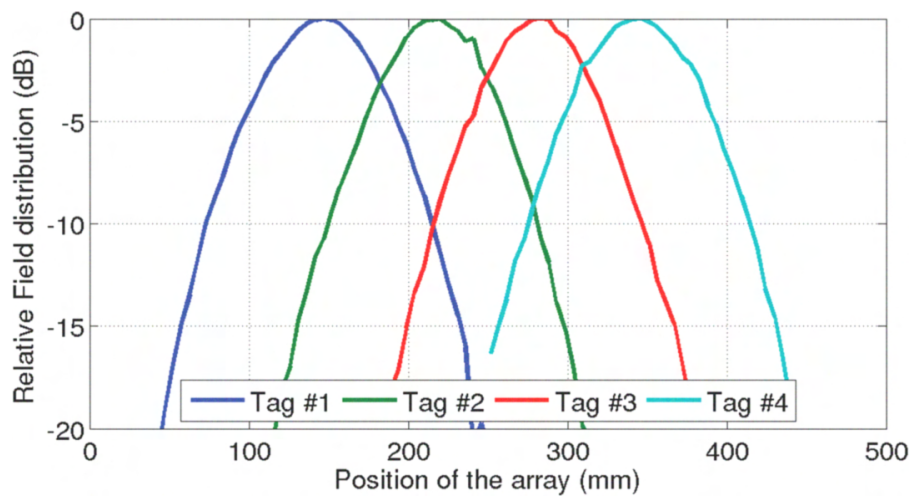

Figure 4: Measured electric field distribution by an array of RFID probes 


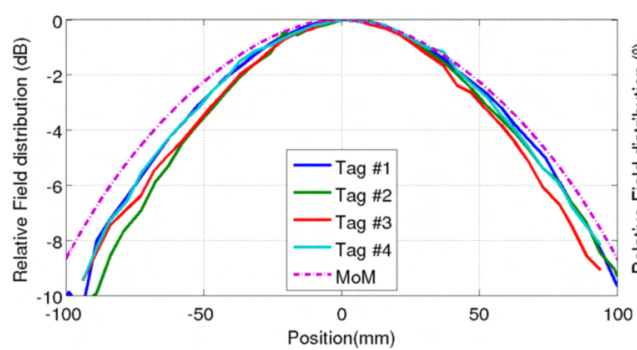

(a) Magnitude

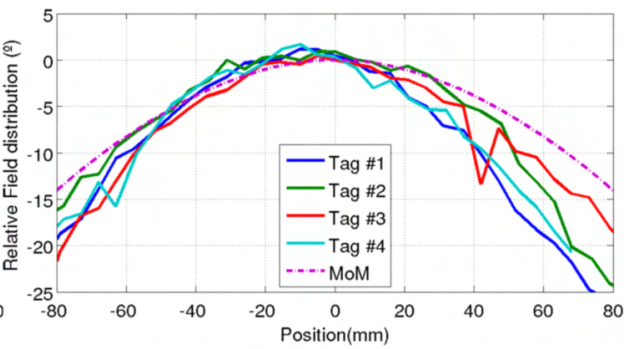

(b) Phase

Figure 5: Measured electric field distribution versus simulated results by MoM

\section{Conclusions}

A complete measurement using an array of RFID tags for EM-field measurement has been introduced and proved as a low-cost solution for EM probing. The measured field distribution obtained from each of the tags forming the array presents a good agreement between each other, and also with respect to the expected field distribution obtained through MoM. The powerless nature of the array makes it a promising array probe that could be used in many applications requiring the measure of a field distribution. Further investigation needs to be done in the calibration procedure to use the complete set of information from the tags to obtain relative field distribution between tags; also the dynamic range and the sensitivity of the measurements need to be further investigated.

\section{Acknowledgments}

This work was supported in part by the Spanish Interministerial Commission on Science and Technology (CICYT) under projects TEC2007-66698-C04-01/TCM and CONSOLIDER CSD2008-00068 and by the "Ministerio de Educación y Ciencia" through the FPU fellowship program.

\section{References}

[1] J.-C. Bolomey and F. E. Gardiol, Engineering Applications of the Modulated Scatterer Technique, A. House, Ed. Artech House, 2001.

[2] J. C. Bolomey, L. Jofre, and S. Capdevila, "Reciprocity-based formulation of RFID tag response in arbitrary environments," in Internation Symposium on Antennas and Propagation (ISAP 08), 2008.

[3] S. Capdevila, M. M. Massud, R. Serrano, A. Aguasca, S. Blanch, J. Romeu, , J. Bolomey, and L. Jofre, "RFID based probes for EM-eld measurements," in EuCAP 2009, 2009. 